International Journal of Pattern Recognition and Artificial Intelligence

Vol. 32, No. 5 (2018) 1850015 (19 pages)

(c) The Author(s)

DOI: $10.1142 /$ S0218001418500155

\title{
Millimeter-Wave Radar and Machine Vision-Based Lane Recognition
}

\author{
Wei Li \\ Intelligent Robot Technology \\ Soochow University, Suzhou, P. R. China \\ lunwen124@163.com \\ Yue Guan \\ Mechanical Engineering \\ Soochow University, Suzhou, P. R. China \\ Liguo Chen and Lining Sun \\ Mechanical and Electronic Engineering \\ Harbin Institute of Technology, \\ Haerbin, P. R. China
}

Received 24 May 2017

Accepted 17 August 2017

Published 29 November 2017

\begin{abstract}
Camera can sensor the environment on the lane by extracting the lane lines, but such detection is limited to a short distance with effect of illumination and other factors; radar can detect objects a long distance away but cannot detect the lane conditions. This paper combined machine vision with millimeter-wave radar and extracted the nearby distinct lane line through images; at the same time, the radar obtained the motion trajectory information of distant vehicles, then the least-square method was used to make curve fitting on those motion trajectory information in order to reconstruct the lane line information. Finally, in the stage of fusing two segments of lane lines, the goodness of fit was applied to complete the matching of corresponding lane lines. While, for areas between two segments of lane lines that neither camera or radar can detect, we established a lane model, utilized probabilistic neural network to select the corresponding lane model for matching, and then used approximate mathematics expression according to the selected lane model, thus obtaining the final front road information of current vehicle.
\end{abstract}

Keywords: Lane recognition; radar; camera; combination.

This is an Open Access article published by World Scientific Publishing Company. It is distributed under the terms of the Creative Commons Attribution 4.0 (CC-BY) License. Further distribution of this work is permitted, provided the original work is properly cited. 


\section{Introduction}

Advanced driving assistance system (ADAS) makes use of various sensors on a vehicle to collect the environmental data inside and outside the vehicle, and detects and identifies the target object, so that the driver can perceive possible dangers in advance and the driving security can be improved. Camera can directly reflect the conditions around the vehicle but only detect a short distance due to the effect of illumination, shelter, etc. Automotive radars are mostly millimeter-wave radars which can detect objects at a far distance. ADAS system requires a clear knowledge of the environment around vehicle, therefore, the information of lane line at a distance can be acquired by radar and thus complements with the information of lane line at a short distance obtained by camera.

Recently, most methods that the researchers use to detect lane are based on machine vision, for example, obtaining the marginal information of lane line according to different gradient operator, and then organizing the marginal images into meaningful structure or using road model for matching ${ }^{1,6,9,16}$; utilizing the Hough Transform, B Spline Curves, and so on to extract lane line. ${ }^{12,15}$ What's more, Ref. 10 used Hough transform to detect the lane mark and background reduction method to detect the moving target. And then according to the three normal lane changing conditions, defining variable lane to judge whether the vehicle has ridden across the lane. Another research ${ }^{14}$ focuses on improving the performance of lane detection for curve road by using a linear approximation method. However, this method cannot guarantee real-time requirements. Reference 2 proposed a method of progressive retrieval for screening feature points inside lane to obtain real parameters of lane. This method improves the traditional Hough transform method by extracting the feature point to reconstruct the road model. The detection of lane line by radar needs to rely on the state of target object, such as DECODK, Bosch. ${ }^{8,13}$ There are also examples that combine machine vision with radar for lane recognition, such as Jilin University. ${ }^{5}$ Mobus et al. proposed to estimate the motion state of front vehicle by radar so as to estimate the curvature of front road and realize the recognition of lane line. ${ }^{7}$

Comprehensively considering current lane detection methods, this paper integrated machine vision with millimeter-wave radar and used Hough Transform to extract distinct lane line at a short distance from the images; meanwhile, the radar obtained the information of motion trajectory of distant vehicle, which then received curve fitting through the least-square method, and later the goodness of fit was used to complete the matching of corresponding lane line. For areas between two segments of lane lines that neither camera nor radar can detect, we established a lane model, utilized probabilistic neural network to select the corresponding lane model for matching so as to reconstruct the lane, and finally obtained the front road information of current vehicle. And relevant discussion was made on dynamically recognizing lane line, which takes time to obtain a stable lane line. 


\section{Extraction of Image Lane Lines and Coordinate Transformation}

\subsection{Extraction of image lane lines}

Lane lines on road are often broken in straight lines or partially covered and so on, so the extraction of image lane lines must be adjusted to this condition. On roads, lane lines mostly approximate to straight lines in a short distance. And Hough transformation is a straight-line extraction method based on overall information statistics. The straight line formula can be obtained through accumulating vote to the locations in parameter space corresponding to the image special points and searching for the peak value of parameter space.

Thus, below straight-line equation is selected as the straight-line model to be examined:

$$
y=k x+b .
$$

If the straight line tends to be in vertical direction, then $k \rightarrow \infty$, and the straight line is not able to be described. Thus, the above equation is transformed into polar coordinate:

$$
\rho=x \cos \theta+y \sin \theta
$$

\subsection{Coordinate transformation}

Camera imaging model is a description of projection from three-dimensional world to two-dimensional world, hence, the coordinate system for camera imaging is composed of three parts: image coordinates, camera coordinates, and world coordinates. In this paper, the model for lane lines information needs to be built in world coordinates, thus the lane lines in the image need to be transformed from image coordinate to world coordinate. The relationship between image coordinate and image physics coordinate is

$$
\left|\begin{array}{c}
u \\
v \\
1
\end{array}\right|=\left|\begin{array}{ccc}
\frac{1}{d x} & & u_{0} \\
& \frac{1}{d y} & v_{0} \\
& & 1
\end{array}\right|\left|\begin{array}{l}
x \\
y \\
1
\end{array}\right|
$$

where $u$ and $v$ are pixel coordinates; $d x$ and $d y$ represent the physical sizes of every pixel, respectively, on the $x$-axis and $y$-axis of image physics coordinate; $\left(u_{0}, v_{0}\right)$ is the coordinate of the image physics coordinate's origin when its in image pixel coordinate; $x$ and $y$ are physical coordinates.

The relationship between camera coordinate and image physics coordinate is

$$
z_{c}\left|\begin{array}{c}
x \\
y \\
1
\end{array}\right|=\left|\begin{array}{lll}
f & & \\
& f & \\
& & 1
\end{array}\right| \begin{gathered}
x_{c} \\
y_{c} \\
z_{c} \\
1
\end{gathered} \mid,
$$


W. Li et al.

where the $\left(x_{c}, y_{c}, z_{c}\right)$ is camera coordinate, whose origin is the optical center of camera. The $Z_{c}$ axis coincides with the camera's optical axis; $x_{c}$ axis and $y_{c}$ axis are parallel, respectively, to the $x$-axis and $y$-axis of image physics coordinate; $f$ represents the focal length of camera lens. The relationship between world coordinate and camera coordinate is

$$
\left|\begin{array}{c}
x_{c} \\
y_{c} \\
z_{c} \\
1
\end{array}\right|=\left|\begin{array}{cc}
R & T \\
0^{T} & 1
\end{array}\right|\left|\begin{array}{c}
x_{w} \\
y_{w} \\
z_{w} \\
1
\end{array}\right|,
$$

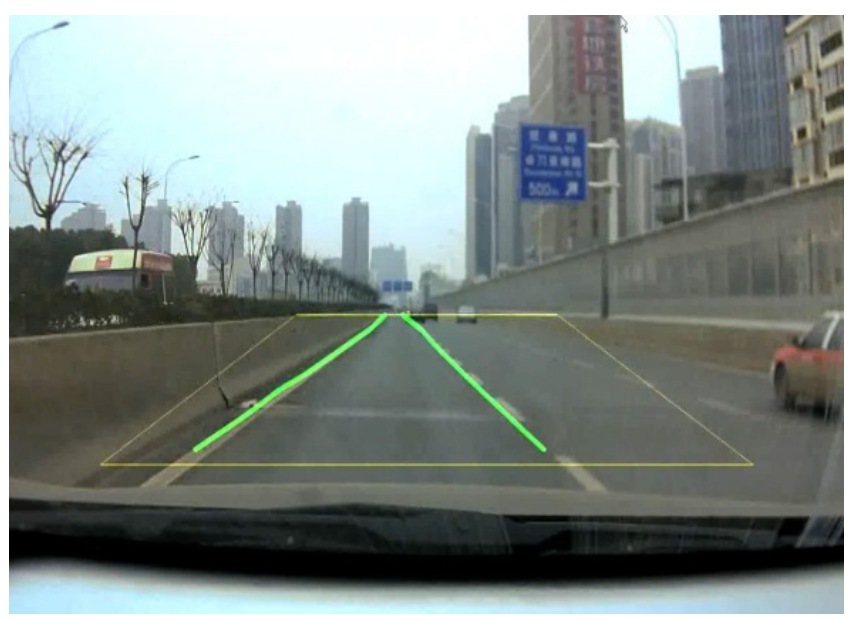

Fig. 1. Camera detection of lane line.

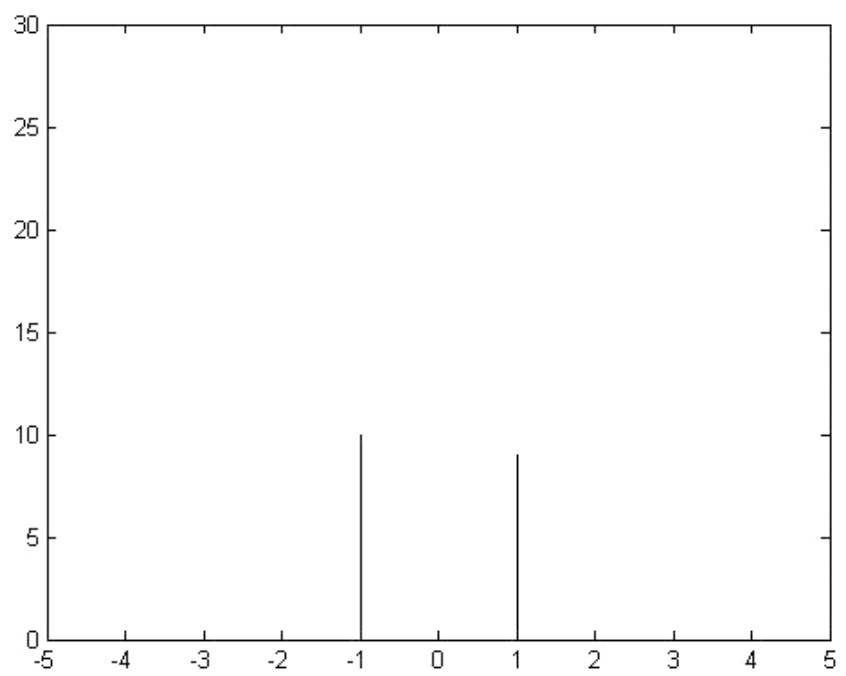

Fig. 2. Lane line coordinate transformation. 
where $R$ is $3 \times 3$ orthogonal unit matrix; $T$ is three-dimensional translation vector; $0^{T}=(0,0,0)$.

From the three equations above, the image coordinate can be revealed in the world coordinate, and the extracted lane lines are displayed in world coordinate as below:

In Fig. 1, the yellow trapezoid area is the range of image lane line detection, and Fig. 2 shows the results of lane line coordinate transformation.

\section{Radar Target Trajectory Tracing}

\subsection{Coordinate compensation}

In this paper, in lane line detection, as both vehicle radar and the target moved in a high speed, the images and the information of lane acquired by radar changed continuously, so all the acquired information of lane line originated from a period of time, and thus the time varying motion needs to be converted into the same coordinate system. However, the information of target acquired by vehicle radar was relative to the coordinate system of current vehicle; before processing the motion trajectory of the target, information detected by radar at a previous moment should be converted into the coordinate system of current vehicle at the current moment, and then the motion trajectory of the target can be processed. Figure 3 is a schematic diagram of coordinate compensation.

It can be seen from the Fig. 3 that, compared to the coordinate of current vehicle at a previous moment, the change of current vehicle at the next moment can be described by rotation transformation and translation transformation. Therefore, the

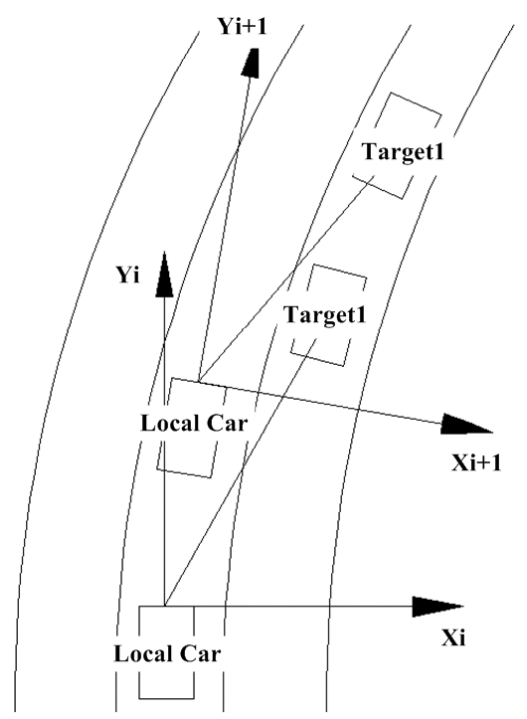

Fig. 3. Coordinate compensation diagram. 
coordinate compensation to the motion of current vehicle can be divided into rotation transformation and translation transformation.

At the moment $i$, the coordinate system of current vehicle can be expressed as $\left(x_{i}, y_{i}\right)$, and the driving speed as $v$. Suppose the sampling period is $T$, and then the distance that current vehicle travels in a sampling period is $L=v \cdot T$. For any one straight line or curve, under certain conditions, it can be approximately deemed as a segment of arc in a circle. This paper used such hypothesis, taking the outline of lane line in a short distance as a segment of arc in a circle. Thereby, the distance is the length of arc that current vehicle travels within time $T$. At the moment $i$, according to the lane line information acquired by camera in current vehicle, we can obtain the radius $r$ of lane line at current moment, and thus, through the arc length formula, the rotation angle of current vehicle can be acquired:

$$
\varphi=\frac{L}{r} .
$$

After rotation, the coordinate state is

$$
\left\{\begin{array}{l}
x_{i}^{\prime}=-x \cos \varphi+y \sin \varphi \\
y_{i}^{\prime}=x \sin \varphi+y \cos \varphi
\end{array}\right.
$$

After translation, the state is approximate to

$$
\left\{\begin{array}{l}
x_{i+1}=x_{i}^{\prime}+L \cos \varphi \\
y_{i+1}=y_{i}^{\prime}+L \sin \varphi
\end{array}\right.
$$

\subsection{Target trajectory curve fitting}

Regarding the motion trajectory of single target, it can be considered as a problem about curve fitting of multiple discrete points. The outline of road is always unfixed, mainly including straight path and various curved paths. It can be known from the model matching-based lane line recognition method ${ }^{4,3}$ that the physical shape of lane line can be fitted by straight line or parabola, which shows the distribution of lane line conforms to the characteristics of mathematical curve to a certain extent. Considering that the targets that radar detects are discrete points and the distribution of front lane line is unknown, this paper uses the Least-Squares method and the B Spline Curves method to fit the trajectory data, respectively.

The curve fitting method based on the principle of minimum sum of deviation squares is called curve-fitting least-square method. The existing analytic equation matched with mathematical laws for linear curve fitting is

$$
y=a_{0}+a_{1} x_{1}+\cdots+a_{p} x_{p}+\varepsilon
$$

where $a_{0}, a_{1}, \ldots, a_{p}$ are unknown parameters, and $\varepsilon$ conforms to $N\left(0, \sigma^{2}\right)$. 
Its curve fitting problem can be transformed into the following equation for solution.

$$
\left\{\begin{array}{l}
\frac{\partial Q}{\partial a_{0}}=-2 \sum_{i=1}^{n}\left[y_{i}-\left(a_{i 0}+a_{i 1} x_{i 1}+\cdots+a_{i p} x_{i p}\right)\right]=0 \\
\frac{\partial Q}{\partial a_{j}}=-2 \sum_{i=1}^{n}\left[y_{i}-\left(a_{i 0}+a_{i 1} x_{i 1}+\cdots+a_{i p} x_{i p}\right)\right] x_{i j}=0,
\end{array}\right.
$$

Through this way, the parameters $a_{0}, a_{1}, \ldots, a_{p}$ are determined.

It is complicated to deal with nonlinear curve fitting directly using the method of least square. However, it can be simplified by describing the estimated parameters in form linear through mathematical transformation and then carrying out the leastsquare curve fitting. Therefore, the method of curve fitting least square can solve most of the problems in curve fitting.

The fitting results aimed at B spline and least square are as follow:

Figure 4 indicates that the curve generated from least-square method matches well with the lane line, whereas the piecewise fitting result of B spline curve is apparently poor than that of least square. Through comprehensive comparison of the two fitting methods, the method of least square is adopted in this paper to progress curve fitting for vehicles' traveling path.

There are certain connections between target trajectory and lane line outline. Use radar to obtain the lane line outline fitting with target trajectory, then with the outline the information of lane line in target place can be rebuilt assuming that the target is traveling on lane all the time. Considering the target's motion trail, multiple targets may exist ahead of the observing car and there may be multiple fitting curves during the process of lane line rebuilding, so deviation will exist in the lane line rebuilt.

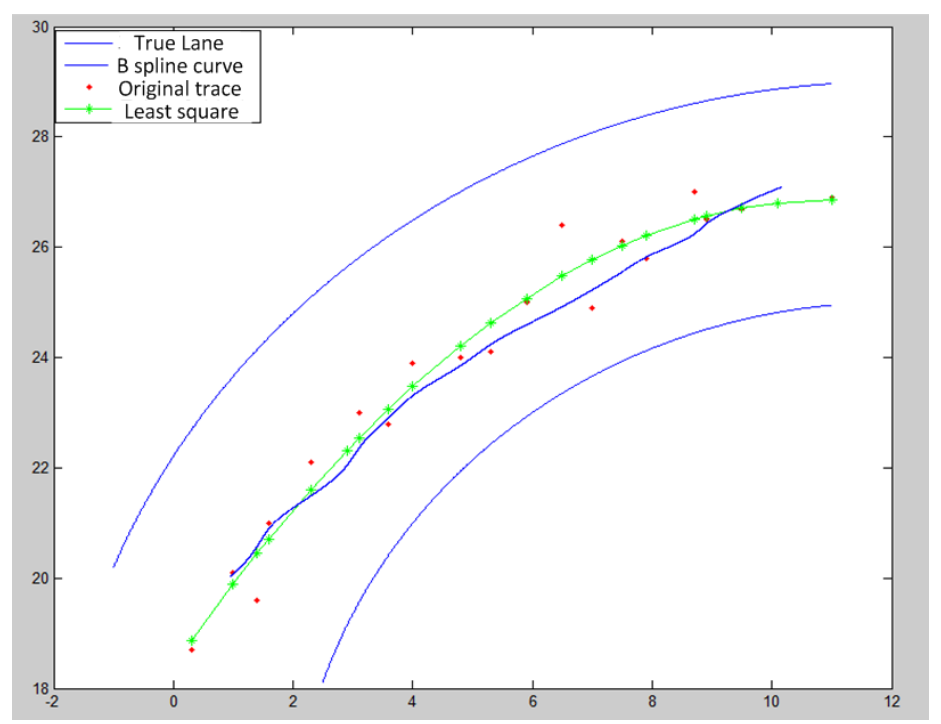

Fig. 4. Comparison of curve fitting results. 

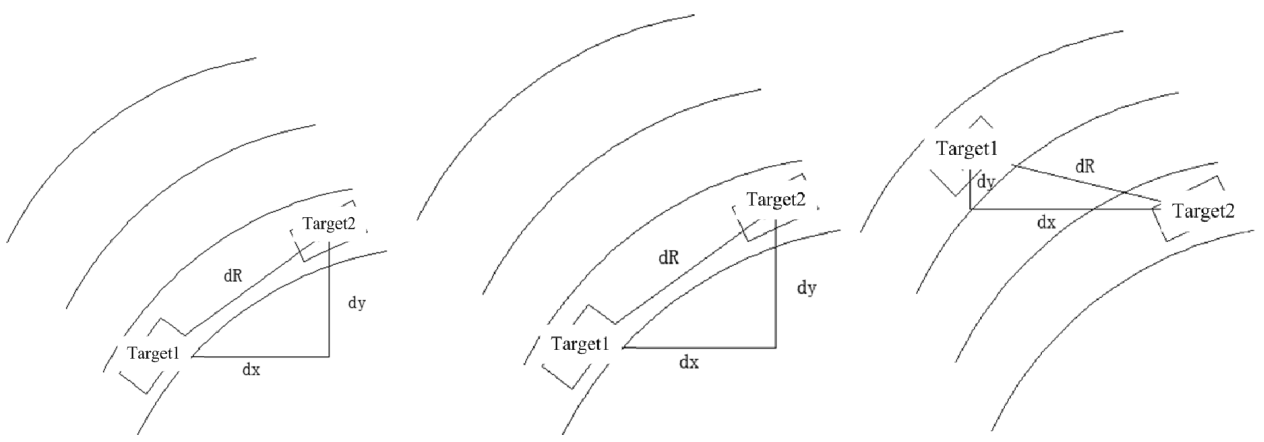

Fig. 5. Schematic diagram of multiple targets.

The fitting lane line outline of a single target's motion trail is nonlinear, and there exist many curvatures on the outline due to fitting errors. Therefore, the rebuilding of target lane line will not rely on obtaining curvatures. In contrast, the fitting lane line outline is used as a template and translated for a certain distance in the target place, which is taken as the practical situation of the lane line in target place.

For multiple targets, only two targets are taken as examples in this discussion, and there are three different conditions as shown in Fig. 5.

It is shown in Fig. 5 that the lane where multiple targets are cannot be confirmed directly assuming that there are three lane lines in the target place. Thus, the method of lane distribution confirmation based on trail fitting is proposed in this paper. The motion trail of each target can be obtained through its historical trajectories, and the lane line outline for each target can also be obtained through curve fitting. By analyzing road shape, it can be known that the road, whether straight or round, can be regarded as a part of a circle in a short distance. Therefore, take the peripheral points of fitting curve as an arc to extend target's motion trail, and the extended curves of all the target trajectories have intersection on the $X$-axis and $Y$-axis of world coordination, then the distance estimation of the intersection area can decide the lane distribution of each target.

As shown in Fig. 6, the black points are target's trail points; the black curve is the fitting lane outline; the points $A, B$, and $C$ are three marginal points on the lane outline. The three points can be described by arc in short distance. Find the circle center $M$, whereby radius $A M=B M=C M$; accordingly, the follow-up trail fitting can predict for the lane line.

As shown in Fig. 7, the red full line is the predicted trail of target 1; the intersection of the trails of target 1 and target 2 is between line $a$ and line $b$; line $c$ is the normal of arbitrary point $\mathrm{E}$ on the predicted trail of target 1, intersecting with the trail of target 2 at point $F$. Set $E$ as $\left(x_{1}, y_{1}\right)$ and $F$ as $\left(x_{2}, y_{2}\right)$, then the distance $R$ between the two curves is

$$
R^{\prime}=\sqrt{\left(x_{1}-x_{2}\right)^{2}+\left(y_{1}-y_{2}\right)^{2}} .
$$




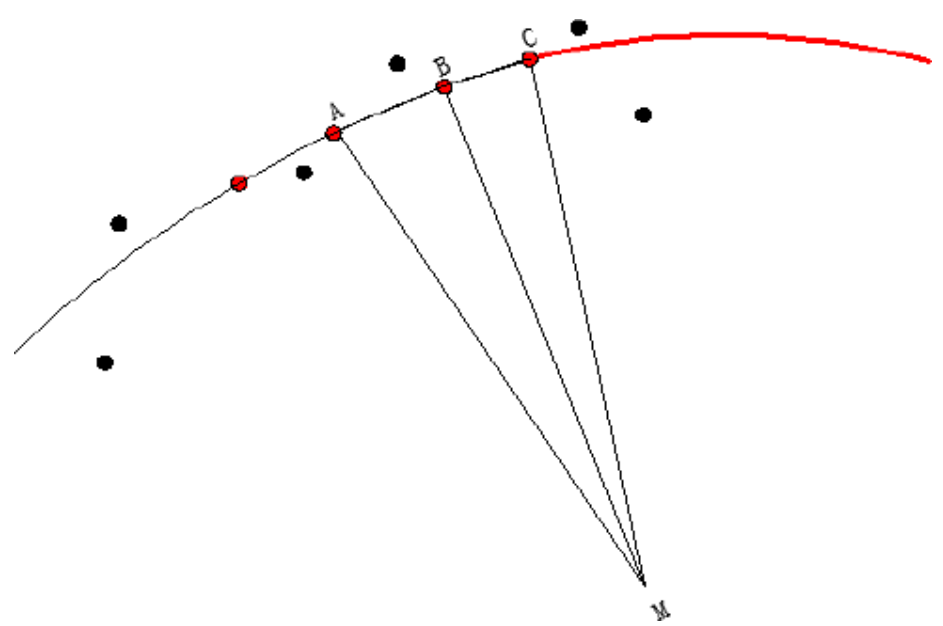

Fig. 6. Lane line prediction method.

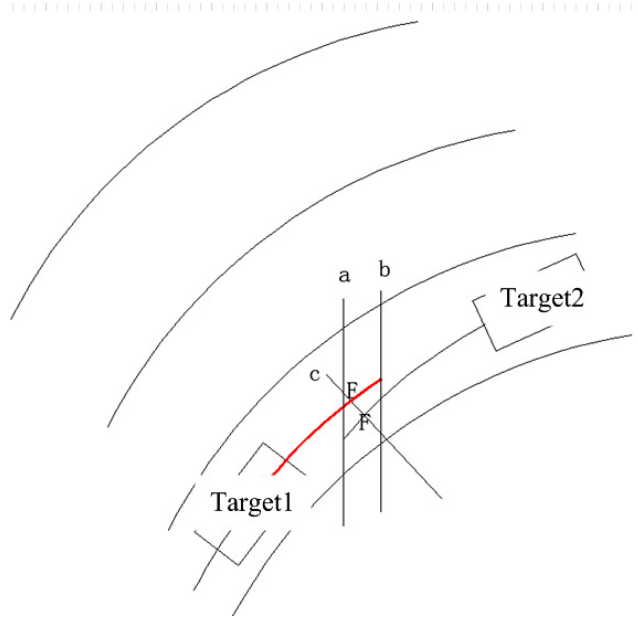

Fig. 7. Schematic diagram of lane line distribution method.

Set the lane width as $H$, then it can be concluded that

when $R^{\prime}<=\frac{H}{2}$, it can be judged that the two vehicles are on the same lane; when $\frac{H}{2}<R^{\prime}<\frac{3 H}{2}$, the two vehicles are on adjacent lanes; when $\frac{3 H}{2},<R^{\prime}<\frac{5 H}{2}$ there is a vacant lane between the two vehicles.

\section{Lane Information Reconstruction}

\subsection{Matching of corresponding lane line}

In order to predict the information of lane in a blank part between the lane lines obtained by camera and radar, first, it is necessary to establish the correspondence 
between the lane lines recognized through images and detected by radar. In this paper, the edge curvature of lane and the goodness of fit are used for matching.

The degree of fitting goodness of fit refers to the degree how regression line fits to the observed value, and the statistics to measure the goodness of fit are coefficient of determination $R^{2}$, whose value range is $[0,1]$. The closer it is to 1 , the better the degree of fitting will be. The curvature of the edge of lane line acquired by camera is calculated as $c_{0}$, which then is used to fit the information of front lane; and the fit lane curve is $l_{0}$; the curvature of the edge of lane line acquired by camera is calculated as $l_{1}$, which then is used to fit the information of nearby lane to get the fitting lane curve $c_{1}$. If $l_{0}$ and $l_{1}$ are fit to the same lane line, the goodness of fit between these two lane lines will be very high. But if that is not a same lane line, its degree of fitting will be low. This paper utilizes $R^{2}$ to measure the similarity of lane lines fit by camera and radar, respectively. Suppose the threshold value of goodness of fit to be $a$. When $a<R^{2}<1$ exists, it can be determined that the current vehicle and the target vehicle are located in the same type of lane, otherwise, they are located in different types of lanes.

As shown in Fig. 8, the information near this segment of lane line of current vehicle is obtained by camera, and the lane line at target position is obtained by radar data, while the middle dotted line needs to be predicted from the information of lane line obtained by camera and radar. By means of above methods, the lane lines fit by using the nearby and distant lane line information, respectively, are the blackened full line is then each lane line is matched and judged by calculating the goodness of fit via $R^{2}$.

The calculation of $R^{2}$ is as below. For the predicted lane line, perform uniform sampling according to $x$-axis coordinate. The lane line value obtained by camera is $y_{i}$, and the lane line value obtained by radar is $Y_{i}$, then the quotients of the regression sum of squares and the sum of squares is $R^{2}$, namely,

$$
R^{2}=\frac{\sum_{i=1}^{n} y_{i}^{2}-\sum_{i=1}^{n}\left(y_{i}-Y_{i}\right)^{2}}{\sum_{i=1}^{n} y_{i}^{2}} .
$$
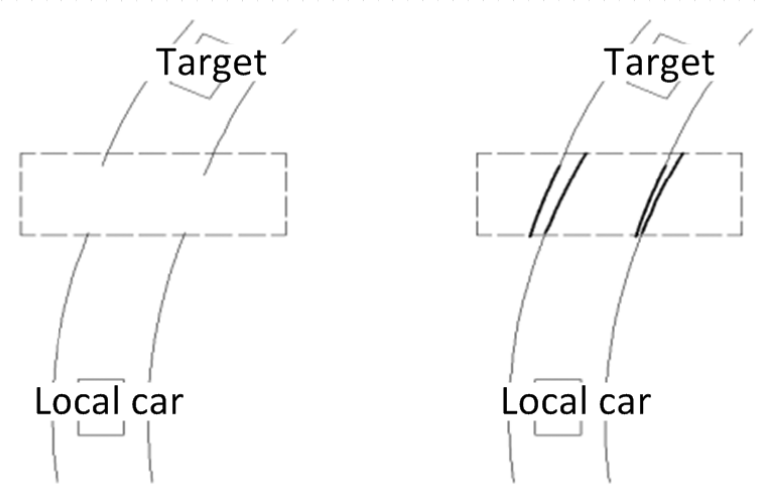

Fig. 8. Fused segment of lane line prediction. 
The lane line conforming to the required degree of fitting is considered to be the corresponding lane line, which is used for reconstructing lane line later.

\subsection{Selection of lane line model}

Investigating the existing conditions of lane lines, there are basically five types as below.

By observing these five types of lane lines, it can be discovered that their differences are the change of tangential slope of lane line at the entrance and exit of lane. Figure 9 (a) is a straight path, and the tangential slope of each point at lane line is infinite; Fig. 9(b) is a right-turn right-angle curve, whose tangential slope reduces from infinite to zero; Fig. 9(c) is a common right-turn curve, whose tangential slope changes between the tangential slopes of two points at the entrance and exit of lane; Fig. $9(\mathrm{~d})$ is a common left-turn curve, whose tangential slope also changes between the tangential slopes of two points at the entrance and exit of lane; Fig. 9(e) is a leftturn right-angle curve, the tangential slope of which changes from minus infinity to zero. Hence, the change of tangential slope can be taken as the characteristic to classify these five types of lane patterns. In order to simplify the calculation of tangential slope, two continuous points on the edge of two segments of lane lines can be directly selected to calculate the slope, as tangential slope.

The probabilistic neural network ${ }^{11}$ is a special artificial neural network used for solving the classification problem, the theoretical basis of which is the Bayes minimum risk rule. The probabilistic neural network places the Bayes estimation in a feedforward neural network, and makes Bayesian decision according to nonparameter estimation of probability density so as to obtain a classification result. The probabilistic neural network has four layers: input layer, hidden layer, summation layer, and output layer, as shown in Fig. 10.

The first layer is input layer, which is used to receive the values from training samples and transmit data to the hidden layer; the number of neuron equals the length of input vector.

The second layer is a hidden layer, also a radial basis layer, which employs the gaussian function as transfer function, with each neuron node having one center. This layer receives the sample data of input layer, figures out the distance between input vector and the center, and finally returns a scalar value. The number of neuron

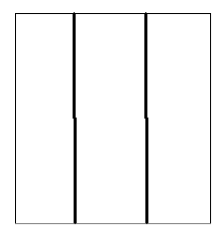

(a)

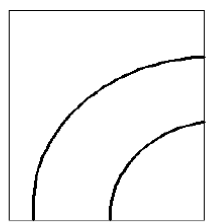

(b)

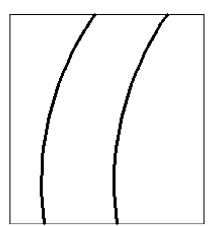

(c)

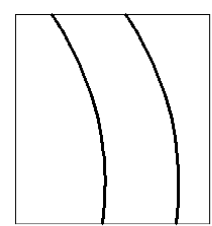

(d)

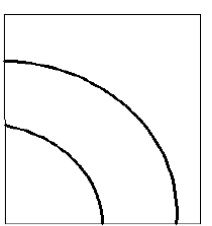

(e)

Fig. 9. Different road models. 
W. Li et al.

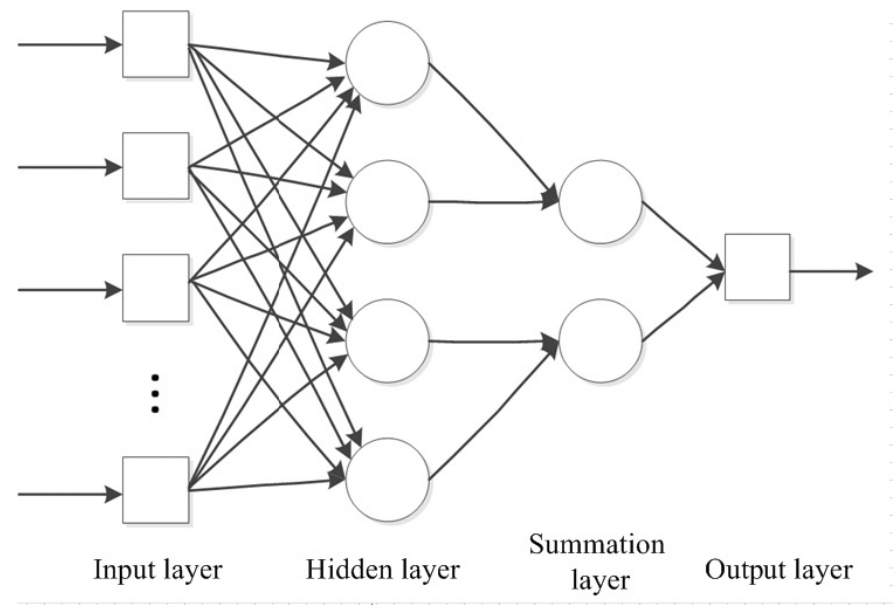

Fig. 10. The structure of probabilistic neural network.

equals the number of input training samples. Inputting vectors into the hidden layer, the input and output relation determined by the $j$ neuron of the $i$ kinds of pattern in hidden layer is defined by the formula below:

$$
\emptyset_{i j}(x)=\frac{1}{(2 \pi)^{\frac{1}{2}} \sigma^{d}} e^{\frac{\left(x-x_{i j}\right)\left(x-x_{i j}\right) T}{\sigma^{2}}},
$$

where $M$ is the total number of category in training samples, $d$ is the number of dimension of sampling space data, $x_{i j}$ is the $j$ center of $i$ kind of sample. This paper chose three samples from each kind of lane models, respectively, for training; therefore, the hidden layer contains 15 neurons.

The third layer is the summation layer, which makes a weighted average on the output of hidden neurons that belong to the same kind in hidden layer:

$$
v_{i}=\frac{\sum_{j=1}^{L} \varnothing_{i j}}{L},
$$

where $v_{i}$ represents the output of $i$ category, $L$ refers to the number of $i$ kind of neurons. In summation layer, the number of neurons is the same as category number $M$, i.e. 5 .

The fourth layer is output layer, which takes the maximum one in summation layer as the output category

$$
y=\arg \max \left(v_{i}\right) .
$$

In actual calculation, the vector of input layer is firstly multiplied with weighting coefficient, and then input into the radial basis function for calculation

$$
Z_{i}=x w_{i}
$$


Suppose $x$ and $w$ have been standardized as unit length, and then conduct radial basis operation on the results, which is equivalent to formula below:

$$
\exp \left(\left(z_{i}-1\right) / \sigma^{2}\right)
$$

which is equal to

$$
\exp \left[-\frac{\left(w_{i}-x\right)^{T}\left(W_{i}-x\right)}{2 \sigma^{2}}\right]
$$

where, $\sigma$ is the smooth factor, the value of which decides the width of the bell-shaped curve taking sample point as a center and thus plays a vital role in the performance of network. Besides, when it varies in a certain range, there will be a value that provides the best recognition effect. Generally, such a value is manually determined, so that a value with the best result can be found in repeated experiments.

As a result, the structure of probabilistic neural network used in this paper is determined to be 2-15-5-5. Regarding the fitting of single lane line, there are two input characteristics for each probabilistic neural network: one is the tangential slope at the endpoint of lane line detected by images, and the other one is the tangential slope at the endpoint of lane line obtained by tracking target through radar. The output is one of the five lane models. The classification results of using probabilistic neural network for testing are shown above in Table 1.

Among these 100 experiments, 98 experiments are correct, with only two "false". So it proves that the correct rate is very high of using probabilistic neural network in lane model for classification distinguishing, which satisfies the need of this paper.

\subsection{Reconstruction of lane line}

After classifying the lane lines via probabilistic neural network, different lane models correspond to different mathematical expression, thereby different lane models shall adopt different reconstruction strategies.

For model (a), the vehicle is located in a straight lane, at this time, we only have to conduct weighted fusion on the lane lines detected by radar and camera.

Table 1. Classification results of neural network.

\begin{tabular}{lcccc}
\hline Sample S/N & Actual Category & Judged Category & Correct/False & Type of Lane \\
\hline 1 & $\mathrm{a}$ & $\mathrm{a}$ & Correct & Straight line \\
2 & $\mathrm{c}$ & $\mathrm{c}$ & Correct & General right-turn curve \\
3 & $\mathrm{~b}$ & $\mathrm{~b}$ & Correct & Right-turn right-angle curve \\
4 & $\mathrm{e}$ & $\mathrm{e}$ & Correct & Left-turn right-angle curve \\
5 & $\mathrm{~d}$ & $\mathrm{~d}$ & Correct & General left-turn curve \\
6 & $\mathrm{~b}$ & $\mathrm{~b}$ & Correct & Right-turn right-angle curve \\
$\vdots$ & $\vdots$ & $\vdots$ & $\vdots$ & $\vdots$ \\
100 & $\mathrm{e}$ & $\mathrm{e}$ & Correct & Left-turn right-angle curve \\
\hline
\end{tabular}


W. Li et al.

For models (b) and (e), the vehicle is located in a right-angle curve. This segment can be approximately considered as an arc, whose coordinate should satisfy

$$
(x+a)^{2}+(y+b)^{2}=r^{2} .
$$

Its first-order derivative is

$$
y^{\prime}=\frac{-(x+a)}{y+b},
$$

where $y^{\prime}$ is the tangential slope. Substituting the tangential slope and coordinate point of the edge of two segments of lane lines, we can obtain the mathematical expression of arc, and directly make curve fitting.

For models (c) and (d), the vehicle is located in a general left-turn or right-turn curve. This lane line is extremely similar to the trajectory of hyperbolic curve with its focus being in the $\mathrm{x}$ axis, so hyperbolic curve can be used for fitting it. Its mathematical expression is

$$
\frac{x^{2}}{a^{2}}-\frac{y^{2}}{b^{2}}=1
$$

Its first-order derivative is

$$
y^{\prime}=\frac{b^{2}}{2 y}\left(\frac{2 x}{a^{2}}-1\right) .
$$

This paper recorded the data of a real vehicle, and took straight lane and common curve that mostly appear on the road as examples for experimental verification. Below are the experimental results according to the methods mentioned in this paper.

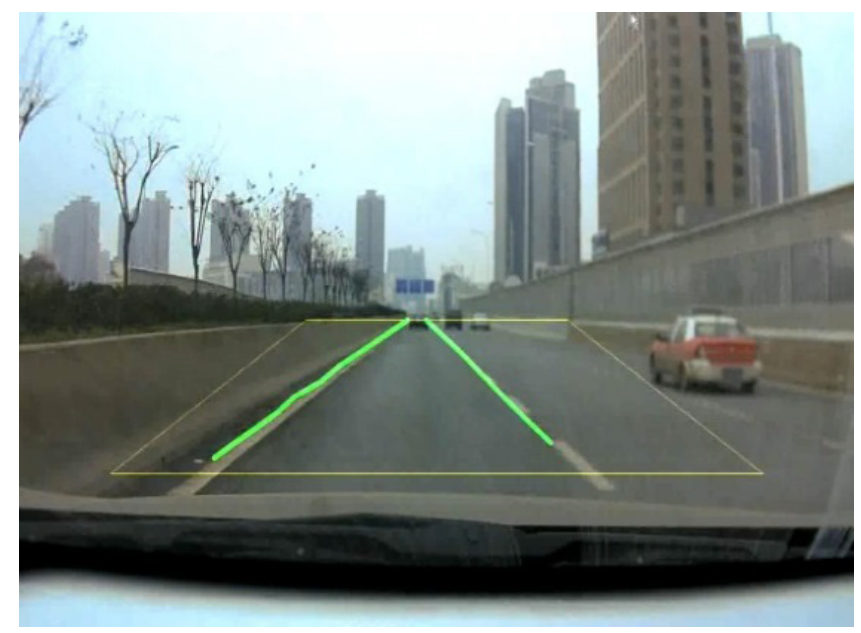

Fig. 11. (Color online) Detection of straight lane. 


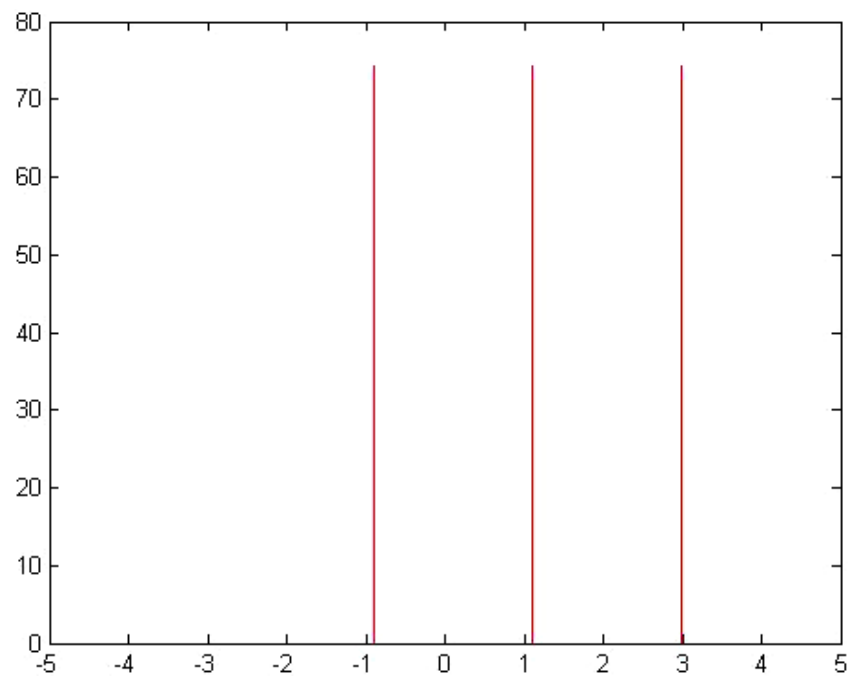

Fig. 12. Reconstruction of straight lane.

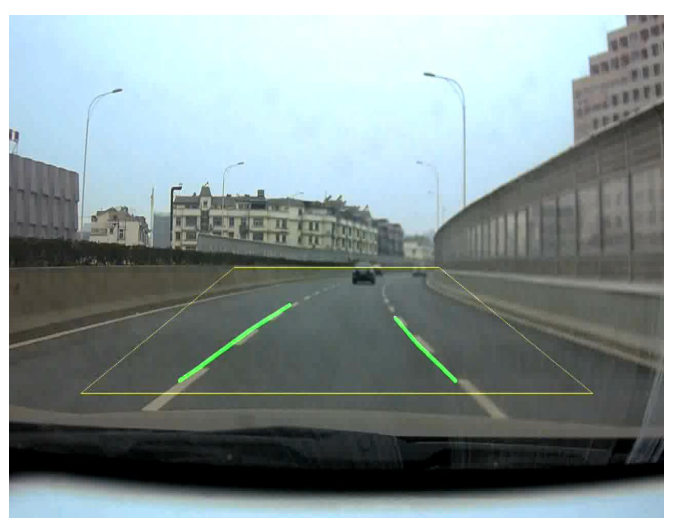

Fig. 13. Detection of general right-turn lane.

Model (a): straight lane, with the initial frame of images and the recognition effect of its lane lines as below.

In Fig. 11, the yellow trapezoid box is the region for lane detection, and the green lines are the results of lane detection. Figure 12 shows the results of transformation.

Model (c): general right-turn curve, with the initial frame of images and the recognition effect of its lane lines as Figs. 13 and 14.

It can be discovered from the experimental results that the method proposed in this paper maintains the change features of lane line as a whole, with a smooth transition from straight lane to curve and a large similarity between reconstructed lane model and real lane. But this method depends on images to recognize the nearby lane lines. Because the Hough Transform has a high accuracy in detection of straight 
W. Li et al.

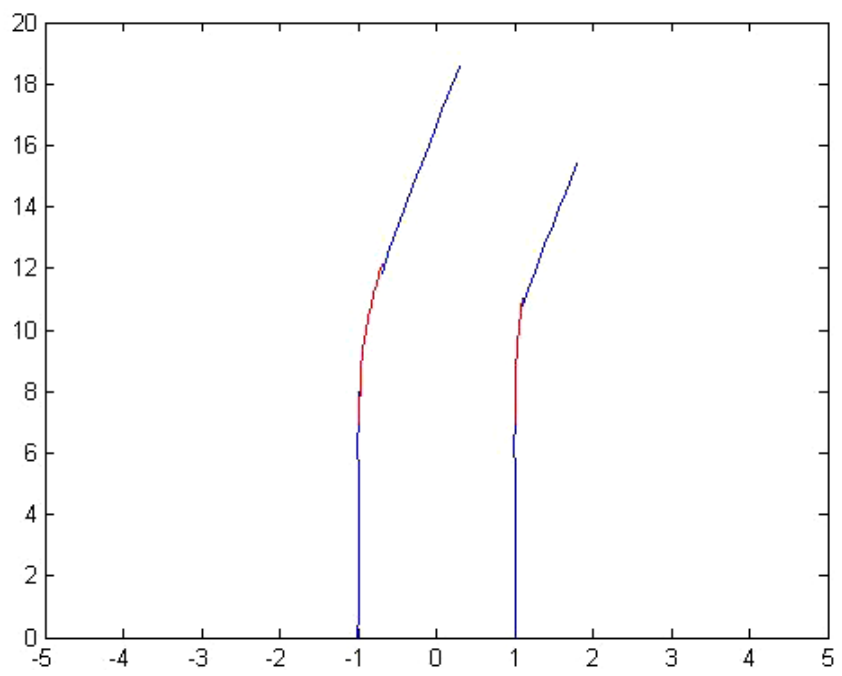

Fig. 14. Reconstruction of general right-turn lane.

line, this results in that even when the vehicle is located in a curve, the lane line will be detected as straight lines from images. The integral experimental results prove the effectiveness of the method proposed in this paper.

\section{Dynamic Recognition}

The above paragraphs discussed the use of historic information of previous period for recognizing and reconstructing the front lane line. But in practical course of traveling, the recognition of lane line is a dynamic course. If the time period required for recognizing lane line once is $t$, to guarantee the consistency and real time of lane line, the time interval between lane recognition can be set as $\frac{t}{2}$. In this way, in two neighboring recognition of lane line, the data collected in the middle time period of $\frac{t}{2}$ will be the same, which ensures the consistency of overall lane line recognition. Regarding the course of dynamic recognition of lane line, there are two points to discuss:

(1) The detection model of two continuous segments of lane lines keeps consistent. In such case, within the time of $\frac{3 t}{2}$, because the lane model remains unchanged, it can be judged that the situation of lane that the vehicle is located in does not change, so we only have to directly make overall weighted average and fitting on these two segments of lane lines.

(2) When recognizing the next segment of lane line, the selected lane line model changes. In such case, the lane lines do change, for example, from straight lane to curve. This paper takes the change from straight lane model to a general right-turn lane model as an example to discuss. Assume conducting the first lane recognition at time point $t$; at this moment, both current vehicle and target vehicle are in the straight lane; moving to time point $\frac{3 t}{2}$, it is time to conduct the second lane 
recognition; at this moment, the lane model of target vehicle is detected to change to a general right-turn curve model. The data of lane recognition at time point $\frac{3 t}{2}$ are the images acquired by camera at time point $\frac{3 t}{2}$ and the motion trajectory data of target acquired by radar at time period $\frac{t}{2}-\frac{3 t}{2}$. As the lane line would not have shape change, so the results of direct lane recognition can reflect the condition of lane line in current situation. However, there will be a deviation between the straight lane line recognized at time point $t$ and that recognized at time point $\frac{3 t}{2}$. Because the information of lane line acquired by camera is more accurate, it is feasible to utilize the information of lane line acquired by camera at time point to make weighting adjustment on the information of straight lane, while the adjusted information of straight lane is taken as the lane information acquired by camera, which receives lane line recognition together with the target trajectory information within time period $\frac{t}{2}-\frac{3 t}{2}$. For other types of model transformation, such a method is also workable. The experimental results of dynamic fitting of this segment of lane line are shown in Figs. 15-18.

In the fitting diagram, the blackened full line refers to the results of fitting adjustment of lane lines at time point $\frac{3 t}{2}$, and the fine full line refers to the results of lane detection at time point $t$. It can be observed from experimental results that when the detection of lane model changes, using the dynamic fitting of lane lines mentioned in this paper can make the recognition of lane line more accurate.

It can be seen from above analysis that, regarding the fitting of a segment of lane line, it is required to conduct the first fitting on historic data at time period $t$, thereby obtaining the whole information of front lane lines. While in the second recognition of

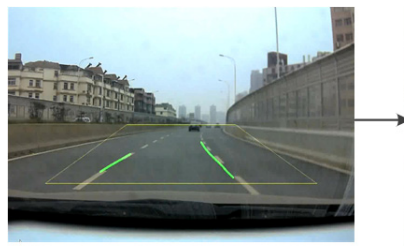

Time $t$

Fig. 15. Initial frame of straight lane-curve.
Time $\mathrm{t}$

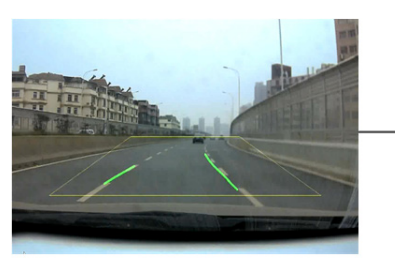

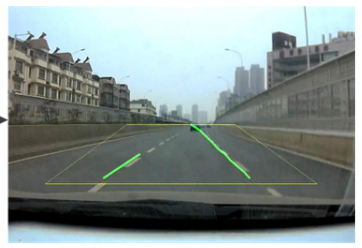

Time 3t/2

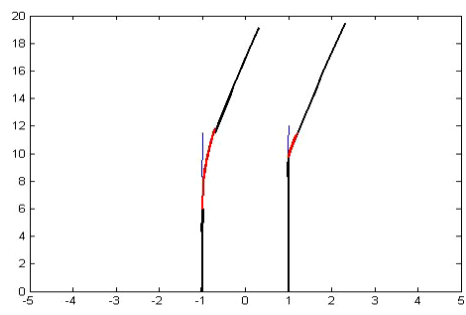

Fig. 16. Comparison of straight lanecurve dynamic fitting.

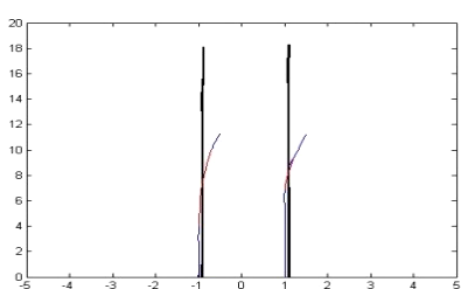

Fig. 18. Comparison of straight lanecurve dynamic fitting. 
lane lines, it is required to adjust the first segment of lane lines and thus we need $\frac{t}{2}$ time at this time. As a result, if we want to obtain a stable information of lane line at front road, we require $\frac{3 t}{2}$ time.

\section{Conclusions}

This paper complements the advantages of radar and camera, and reconstructs the front lane line of vehicle. Through the images near the vehicle acquired by camera, we can extract the information of nearby lane line and convert them into world coordinates; while the motion trajectory information of distant target acquired by radar receives curve fitting through the least-square method; finally, in the fusion segment, this paper utilizes the degree of fitting goodness of fit as the criterion of matching the corresponding lane lines, selects the corresponding road model via probabilistic neural network, establishes different mathematical expression according to different road models for reconstructing lane lines, and obtains the ultimate front road information of current vehicle. At last, the paper discusses the method of dynamic recognition of lane lines and confirms the time required for acquiring accurate lane lines. Compared with single camera, this method can detect farther distance; compared with single vehicle radar, this method is more stable. The experimental results indicate that the method proposed in the paper is effective, but relies on the motion trajectory of target vehicle and so needs further improvement.

\section{References}

1. A. Broggi, Robust real-time lane and road detection in critical shadow conditions, in Proc. Int. Symp. Computer Vision, Coral Gables (IEEE, 1995), pp. 353-358.

2. J. Duan, G. Liu and B. Zhang, Algorithm of fast lane identification based on overall optimal threshold, J. Beijing Univ. Technol 41(1) (2015) 42-47.

3. G. Lin, X. Chen and W. Zhang, Multi-information fusion optimization based robustness lane detection algorithm, J. Southeast Univ. Nat. Sci. 40(4) (2010) 771-777.

4. A. Lopez, J. Serrat, C. Canero et al., Robust lane markings detection and road geometry compution, Int. J. Automot. Technol. 11(3) (2010) 395-407.

5. S. Lu, Study on Lane Detection Using Vision and Radar (Jilin University, 2015), p. 6.

6. F. Paetzold, U. Pranke, W. Von Seelen et al., Lane recognition in urban environment using optimal control theory, Intelligent Vehicles Symp., Dearborn (IEEE, 2000), pp. 221-226.

7. M. Rainer, B. Mato and M. Manfred, Multi-Object Adaptive Cruise Control (Springer Berlin Heidelberg, Berlin 2003).

8. J. K. Schiffmann and G. R Wilamann, Model-based scene tracking using radar sensors for intelligent automotive vehicles systems, in IEEE Conference on Intelligent Transportation System, 1997/ITSC'97 (IEEE, 1997), pp. 421-426.

9. H. Shen, S. Li, F. Bai et al., Monocular vision detection method of lane line in structured road, Chin. J. Sci. Instrum. 31(2) (2010) 397-403.

10. Y. Shi, J. Deng and H. Luo, Vehicle lane change track recognition method based on video detection, Technol. Econ. Areas Commun. 19(3) (2017) 48-53. 
11. L. Su and X. Song, Realization and application of probabilistic neural network based on Matlab, Comput. Modern. 11 (2011) 47-50.

12. B. Tian, B. Zheng and $\mathrm{Q}$. Wu, Research on expressway lane detection and tracking algorithm, Intell. Transport 2008(9) 180-183.

13. W. Uhler, M. Scherl and B. Lichtenberg, Driving course prediction using distance sensor data, in Int. Congress and Exposition, Detroit, Michigan, March 1-4 (1999), pp. 127-135.

14. B. Wang, Z. Qi and G. Ma et al., Novel lane curve detection method using linear approximation, Trans. Beijing Inst. Technol 36(5) (2016) 470-474.

15. Y. Wang, E. K. Teoh and D. Shen, Lane detection and tracking using B-snake, Image Vis. Comput. 22(4) (2004) 269-280.

16. B. Yu and A. K. Jain, Lane boundary detection using a multiresolution hough transform, Int. Conf. Image Processing, Washington, DC (IEEE, 1997), pp. 748-751.

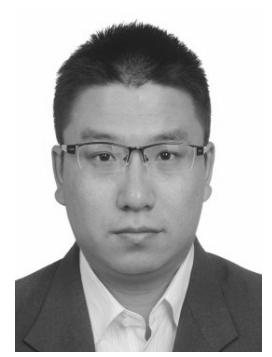

Wei Li is a Ph.D. candidate at the Soochow University. $\mathrm{He}$ is also a senior engineer and won the Shanghai Science and Technology Progress Award, as well as the honor of Shanghai Science and Technology Pioneer. His research is on automotive-electronic products such as radio frequency and vehicle sensors. He made great achievements in the technical development of radio frequency, vehicle sensors, ADAS, and other related products, and has published 3 related papers; he applied for 32 domestic patents and 5 international patents altogether.

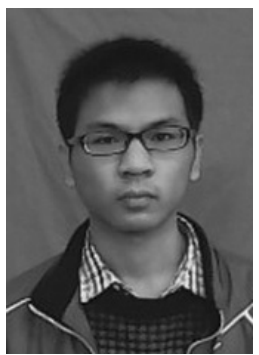

Yue Guan is a masters student at Soochow University. In 2014, he received his bachelor's degree in Engineering from Soochow University. He studied at the School of Mechanical and Electrical Engineering of Soochow University. $\mathrm{He}$ is mainly engaged in the study of vehicle millimeter-wave radar signal processing, and has been engaged in millimeterwave radar research for years; thus he has a profound understanding of millimeter-wave radar applications.

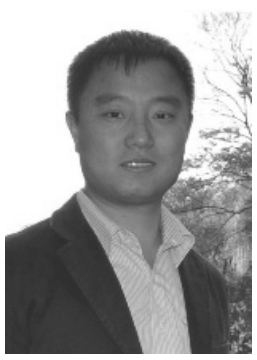

Liguo Chen is a professor and doctoral supervisor. He received his degree in Engineering from Harbin University of Science and Technology in 1997, and his master's degree in Engineering from Harbin University of Science and Technology in 2000. He received his Ph.D. in Engineering from the Harbin Institute of Technology in 2003 and currently, he is a professor of robotics of Microsystem Research Center, School of Mechanical and Electrical Engineering, Soochow University. He is mainly engaged in micro operations and micro drive robot research.

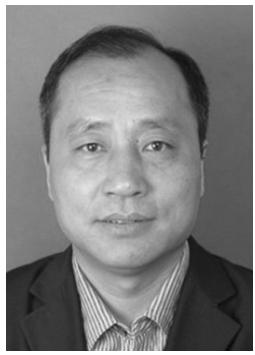

Lining Sun is a professor and doctoral supervisor, and also a distinguished professor as a Yangtze River scholar. He is currently serving at the School of Mechanical and Electrical Engineering, Soochow University as the Dean. He is mainly engaged in the research and development of industrial robots and mechatronics equipment, micro/nano-operating robots and equipment, and medical and special robots. He has published more than 200 papers and won more than 20 national invention patents. 